\title{
Pre- and post-surgery change of EGFR mutation status in a lung squamous cell cancer patient
}

\section{Mingming $\mathrm{Hu}$}

Beijing Chest Hospital

\section{Fugen Li}

Beijing Chest Hospital

Yun Zhao

Chuiyangliu Hospital Beijing

\section{Baohua Lu}

Beijing Chest Hospital

Haifeng Lin

Beijing Chest Hospital

Hongmei Zhang (D 790016548@qq.com )

Capital Medical University

Tongmei Zhang ( $\square$ tongmeibj@163.com )

Beijing Chest Hospital

\section{Case report}

Keywords: lung squamous cell cancer, EGFR, ctDNA, neoadjuvant chemotherapy, surgery, dynamic change

Posted Date: April 1st, 2021

DOl: https://doi.org/10.21203/rs.3.rs-335690/v1

License: (c) (1) This work is licensed under a Creative Commons Attribution 4.0 International License. Read Full License 


\section{Abstract}

\section{Background}

The percentage of epidermal growth factor receptor (EGFR) mutations in squamous lung cancer was relatively low. The dynamic changes of EGFR mutation in peripheral blood before and after surgical resection in epidermal growth factor receptor (EGFR) has been rarely reported.

\section{Case presentation}

We report a 73-year-old woman with squamous lung cancer of the left upper lobe and EGFR exon 19 deletion (19del) was identified in biopsy and peripheral plasma separately before a surgical operation. The mass regressed remarkably after three cycles of platinum-based neoadjuvant chemotherapy and then the patient was administered curative-intent surgery. Postoperative pathology supported the diagnosis of left upper lobe squamous cell carcinoma and EGFR 19 del mutation. At the time of 40 days after surgery, EGFR 19del mutation in peripheral blood was undetectable using amplification refractory mutation system-polymerase chain reaction (ARMS-PCR) and next-generation sequencing (NGS) method.

\section{Conclusions}

We report, for the first time, the perioperational dynamics of EGFR mutation in a LSCC patient. EGFR $19 \mathrm{del}$ mutation was eliminated after surgical operation. The genetic mutation status from peripheral blood could be converted through the operation.

\section{Background}

Lung cancer remains the most common malignancy and the leading cause of cancer-related death worldwide, accounting for nearly $20 \%$ of all cancer mortality[1]. Non-small cell lung cancer (NSCLC) is the most frequent type of lung cancer, accounting for $80-85 \%$ of cases. Some large sample cohorts have reported that the frequency of EGFR mutations is approximately $50-60 \%$ among Asian NSCLC populations [2, 3]. The identification of EGFR mutations defined a special subgroup of NSCLC who could respond to EGFR tyrosine kinase inhibitors (TKIs). Classic EGFR activating mutations, including EGFR exon 19 deletion (19del) and Leu858Arg (L858R) point mutation in exon 21, are associated with sensitivity to EGFR-TKI therapy.

For genomic profiling, testing for EGFR mutation has become routine in clinical management of patients with lung carcinoma. Because of the low frequency of EGFR mutation in LSCC, most studies focus only on adenocarcinomas. Tumor-specific genomic alterations can be identified in circulating tumor DNA (ctDNA) from blood samples, which could be especially useful when tumor tissue is unavailable or insufficient for test in monitoring detection of recurrence and tracking resistance. This is, to the best of our knowledge, the first reported case of EGFR mutation dynamic changes before and after surgical operation monitored through ctDNA in a LSCC patient. 


\section{Case Presentation}

A 73-year-old female nonsmoker presented with cough and left upper limb radiating pain was hospitalized for examination of a community hospital. Tumor marker test on July 22, 2020 revealed: CA125 $24.1 \mathrm{ng} / \mathrm{ml}$ (<35 ng/ml), CA199 $7.6 \mathrm{ng} / \mathrm{ml}(<37 \mathrm{ng} / \mathrm{ml})$, CEA $0.8 \mathrm{ng} / \mathrm{ml}(<10 \mathrm{ng} / \mathrm{ml})$, AFP 2.1 $\mathrm{ng} / \mathrm{ml}(<10 \mathrm{ng} / \mathrm{ml})$, SCC $46.4 \mathrm{ng} / \mathrm{ml}(<1.5 \mathrm{ng} / \mathrm{ml})$. Computed tomography (CT) showed a huge mass of $6.5 \mathrm{~cm} \times 8.5 \mathrm{~cm}$ in left upper lobe and a few slightly enlarged mediastinal lymph nodes (Fig. 1a, b). Positron emission tomography-computed tomography (PET-CT) displayed prominent uptake of fluorine-18fluorodeoxyglucose (FDG) in left lung mass (about 26.2 maximum standardized uptake value [SUVmax]) and moderately increased uptake of mediastinal lymph node (station 4L, SUVmax 7.2, Fig. 1c, d). CTguided percutaneous biopsy findings indicated a squamous cell carcinoma of the left lung mass with immunohistochemistry $(\mathrm{IHC})$ staining (Fig. 3a-c). The diagnosis and stating of mediastinal lymph nodes was obtained by endobronchial ultrasound-guided transbronchial needle aspiration (EBUS-TBNA). Two lymph nodes (stations $4 L, 7$ ) were sampled, but no evidence of malignance was gained. There was no metastases to the abdomen, brain, or bone. Based on the examinations, a tentative diagnosis of LSCC with T4NOMO (stage IIIA, according to the eighth edition of TNM) was proposed. A multiple-gene NGS panel of the biopsy sample revealed the presence of a codon 2235_2249 deletion in EGFR exon 19, with abundance of $26.7 \%$. Also, the ctDNA from plasma was extracted to identify the existence of EGFR 19 del by ARMS-PCR(Fig. 2a, c).

To reduce tumor size with the goal of making it resectable, albumin-bound paclitaxel (total dose of $100 \mathrm{mg} / \mathrm{m} 2$ on days $1,8,15$ ) and Nedaplatin (total dose of $80 \mathrm{mg} / \mathrm{m} 2$ on day $1, \mathrm{~d} 21$ ) were given as neoadjuvant chemotherapy. After 3 cycles of the above therapy, CT revealed that the tumor regressed from $6.5 \mathrm{~cm} \times 8.5 \mathrm{~cm}$ to $4.3 \mathrm{~cm} \times 2.2 \mathrm{~cm}$ in October 2020 (Fig. 1e, f). The therapeutic effect was evaluated as PR according to response evaluation criteria in solid tumors 1.1 (RECIST 1.1). The subsequent wedge resection had been performed in November 2020. Surgical pathology confirmed the diagnosis of LSCC with pleural invasion (3.2cm of diameter maximum). IHC staining of these samples revealed that P40 and CK5/6 were positive, while TTF-1 and NapsinA were negative (Fig. 3d-h). No lymph nodes metastasis was found at the dissected nodes at station $4(0 / 2), 5(0 / 2), 6(0 / 2), 7(0 / 1), 8(0 / 1), 9(0 / 1)$ and $10(0 / 1)$. The surgical specimens also confirmed EGFR 19 del by fluorescence PCR (Fig. 2b). After 30 days for recovery postoperatively, this patient was admitted to our hospital for adjuvant therapy. At that time, EGFR 19del was not detected based on ctDNA from plasma by ARMS-PCR. In order to consolidate the result, a NGS panel including 10 lung cancer-associated genes was performed (Fig. 2d, e). Up to now, It has been 5 months after surgery, the EGFR mutation remained negative, and there is no evidence of recurrence from radiological data.

\section{Discussion}

In the present case, we described a patient with IIIA LSCC accepting the platinum-based doublet chemotherapy as a neoadjuvant targeted therapy was submitted to radical surgery. The EGFR mutation 
dynamics before and after operation was monitored during this period. Several interesting aspects of the case inspire us to make the case report.

Comparing with the high frequency of EGFR gene mutations in Asian lung adenocarcinoma, the percentage of EGFR mutation in LSCC has been reported from 3-8\%[4-6]. Because of its rare incidence, when an EGFR mutation in non-adenocarcinoma was detected, the pathological diagnosis and EGFR testing standards should be consolidated further. In this case, samples of biopsy and operation were both performed by routine histopathologic section and IHC staining to confirm the pathological histology. In a study conducted in Japan, totally, 89 frozen tumor specimens that had been firstly diagnosed as LSCC, were tested for EGFR mutation. The frequency of EGFR mutations was 5.6\% (5/89), but two of five were revised to adenosquamous carcinoma and adenocarcinoma by IHC staining [7]. Here, EGFR mutation for this patient was conducted in samples from biopsy, surgical specimens and peripheral blood, separately. The result derived from NGS made accordance with that from PCR.

Because of eliminating micrometastases and improving the possibility of complete resection, neoadjuvant therapy is supported by surgeons. A large number of randomized controlled trials (RCTs) have shown that neoadjuvant chemotherapy can enhance the 5 -year survival rate of NSCLC by nearly $5 \%$ [8]. EGFR-TKI as neoadjuvant therapy was reported sometimes in recent years, however, there are not enough evidence from large sample RCTs. A meta-analysis with aim to evaluate the efficacy and safety of different neoadjuvant therapies revealed that, only neoadjuvant chemotherapy with platinum displayed a significant advantage in OS, comparing with surgery alone [9]. Therefore, for this patient with EGFR $19 \mathrm{del}$, platinum-based dual-drug chemotherapy was administered followed by curative-intent surgery. A significant decrease in T stage from T4 to T2a was achieved.

Last but not least, ctDNA has been found to contain gene mutations representative of those found in primary tumors [10]. Plus the minimal invasiveness and reproducibility, ctDNA has been the most studied and widely adopted source for EGFR mutations detection and resistance supervising in lung cancer. In this case report, we found EGFR mutation shift in plasma ctDNA before and after surgical tumor resection. To date, limited studies involved the influence of surgical operation on EGFR mutation status. Hu et al. had reported two cases whose EGFR mutation of ctDNA was detected at the time of 30 days after surgery, recurred in 4 months after surgery. As for ctDNA, similar discoveries were found that post surgery ctDNA is a potential predictive biomarker for relapse of lung cancer than the commonly used tumor marker [11-13]. For this patient, EGFR mutation monitoring was conducted every two months through ARMS-PCR. Up to now, it has been 5 months after surgery, and the EGFR mutation remained negative, no evidence of recurrence from radiology found.

\section{Conclusion}

We have presented a LSCC with EGFR 19del who was initially administered for three cycles of platinumbased dual-drug neoadjuvant chemotherapy followed by a significant tumor regression, then a radical tumor resection was conducted for her. What's more, the dynamic EGFR mutation status was tested 
before and after surgery. To the best of my knowledge, this is the first case to observe the dynamic changes of EGFR mutation status during perioperational period in a LSCC patient.

\section{Abbreviations}

EGFR: epidermal growth factor receptor; LSCC: lung squamous cell cancer; ARMS-PCR: mplification refractory mutation system-polymerase chain reaction; NGS: next-generation sequencing; NSCLC: nonsmall cell lung cancer; TKI: tyrosine kinase inhibitors; ctDNA: circulating tumor DNA; CT: Computed tomography; PET-CT: Positron emission tomography-computed tomography; FDG: fluorine-18fluorodeoxyglucose; SUVmax: maximum standardized uptake value; IHC: immunohistochemistry;EBUSTBNA,endobronchial ultrasound-guided transbronchial needle aspiration; TTF-1: thyroid transcription factor-1; CK5/6: Cytokeratin 5 or 6

\section{Declarations}

\section{Acknowledgements}

Not applicable.

\section{Funding}

The authors declare that no funds have been received.

\section{Competing interests}

he authors declare that they have no competing interests.

\section{Consent for publication}

The availability of the data and material concerning the case report was collected during their hospitalization. The data collection had been approved by the institutional ethics board. Written informed consent was obtained from the patient for publication of this case presentation and accompanying images.

\section{Availability of data and materials}

The data used during the current case report is available from the corresponding author on reasonable request.

\section{Authors' contributions}

$\mathrm{MMH}, \mathrm{FGL}, \mathrm{YZ}$ and $\mathrm{HMZ}$ participated in the care of the patient. MMH and TMZ performed the literature review and drafted the manuscript. HFL obtained the pathological data. BHL revised the manuscript. All authors read and approved the final manuscript. 


\section{Author details}

${ }^{1}$ Oncology department, Beijing Chest Hospital of Capital Medical University, Beiguan Street, Tongzhou District, 101149, Beijing, China

${ }^{2}$ Department of Thoracic Surgery, Beijing Chest Hospital of Capital Medical University, Beiguan Street, Tongzhou District, 101149, Beijing, China

${ }^{3}$ Department of Thoracic Surgery, ChuiYangLiu Hospital affiliated to Tsinghua University, Chui yangliu South Street, 100022, Chaoyang District, Beijing, China

${ }^{4}$ Pathology department, Beijing Chest Hospital of Capital Medical University, Beiguan Street, Tongzhou District, 101149, Beijing, China

Ethics approval and consent to participate

The study was approved by the institutional ethics board of Beijing Chest Hospital of Capital Medical University.

\section{References}

The list of citations are not available with this version

\section{Figures}




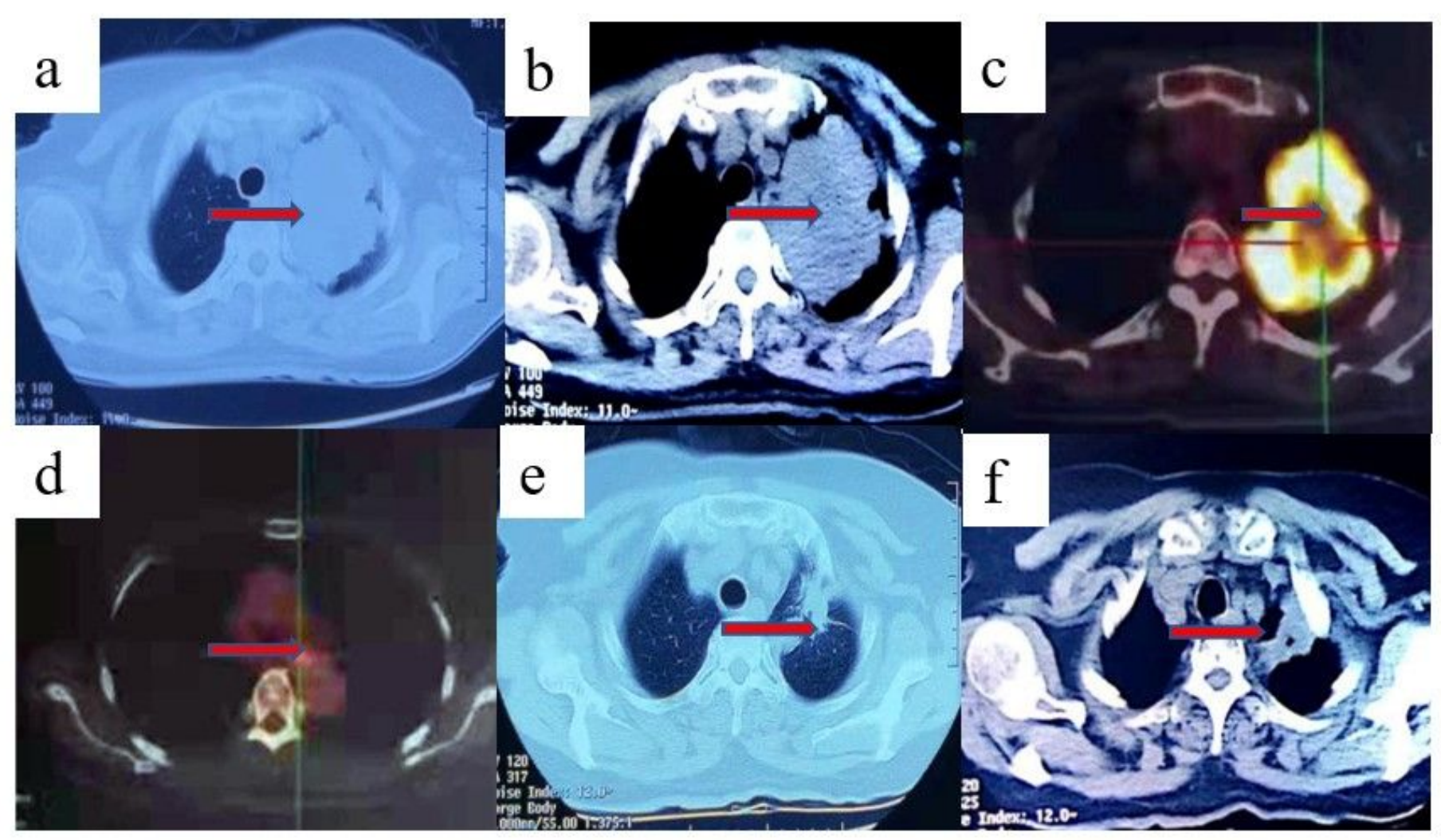

\section{Figure 1}

CT and PET-CT showed a tumor in the left upper lobe. (a-b) CT scan showed a mass in left upper in the lung window and mediastinal window. (c-d) FDG uptake was detected in the left upper lobe and a lymph node at station 4L. (e-f) CT scan showed significant tumor regression in lung window and mediastinal window. 


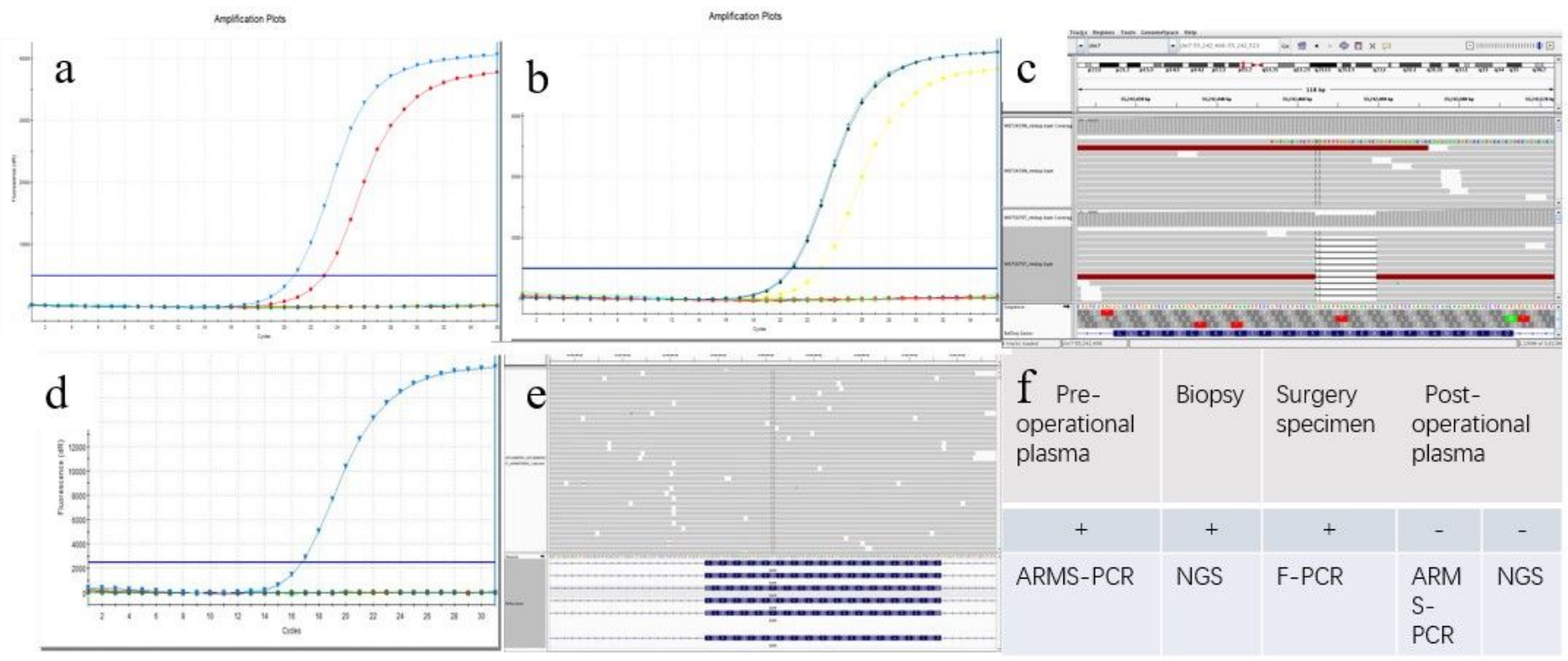

\section{Figure 2}

The EGFR mutation examination before and and after surgical resection.(a) EGFR 19del was identified in peripheral plasma by ARMS-PCR before operation. (b) EGFR 19del was identified in surgical specimen by fluorescence-PCR.(c) EGFR 19del was detected in biopsy before operation with abundance of 26.7\%. (d) EGFR 19del was undetected in peripheral plasma by ARMS-PCR after operation. (e) EGFR 19del was undetected in peripheral plasma by NGS after operation.

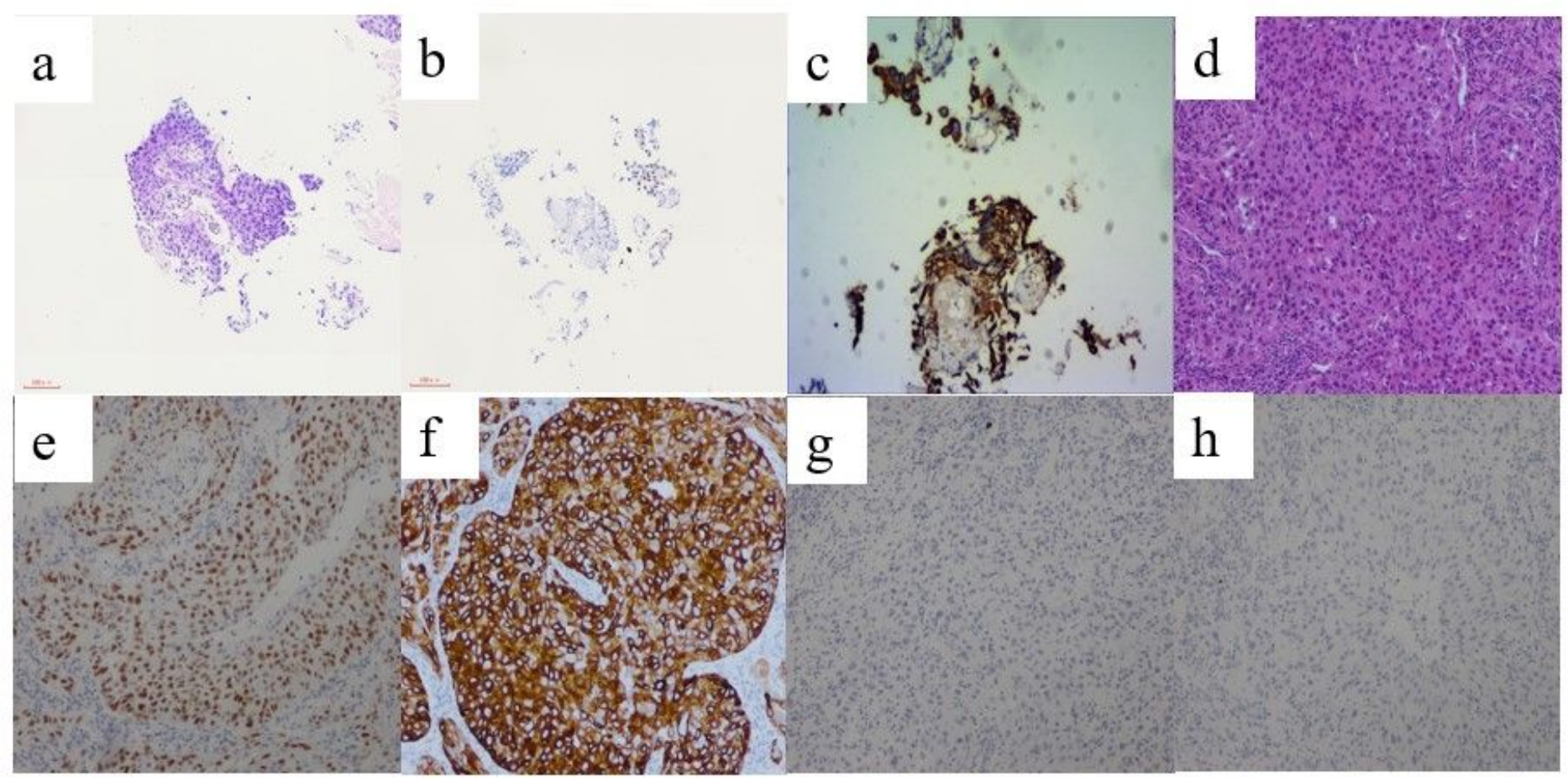

Figure 3 
A.(a-c) Histopathological and immunohistochemical results of CT-guided percutaneous biopsy. (a) H\&E staining (b) P40 (c) CK5/6. (d-h) Histopathological and immunohistochemical results of the surgical specimen. (e) P40 (d) CK5/6 (f) TTF-1 (g) NapsinA. 